Journal Club

Editor's Note: These short, critical reviews of recent papers in the Journal, written exclusively by graduate students or postdoctoral fellows, are intended to summarize the important findings of the paper and provide additional insight and commentary. For more information on the format and purpose of the Journal Club, please see http://www.jneurosci.org/misc/ifa_features.shtml.

\title{
Adolescent Prozac Administration Exerts Antidepressant- Like Effects in Adulthood by Reducing ERK2 Signaling in the
} VTA

\author{
Millie Rincón-Cortés \\ Neuroscience and Physiology, NYU Sackler Institute for Graduate Biomedical Sciences, NYU School of Medicine, New York, New York 10016, and \\ Emotional Brain Institute, Nathan Kline Institute, Orangeburg, New York 10962 \\ Review of Iñiguez et al.
}

Depression is a prevalent and debilitating psychiatric disorder characterized by symptoms such as anhedonia, disrupted social behavior, and alterations in stress-responsive neurobiological systems, which have all been replicated in animal models (Nestler et al., 2002; Ansorge et al., 2007; Nestler and Hyman, 2010; Raineki et al., 2012). The most common age of onset for many psychiatric disorders, including depression, is adolescence; and adolescent onset is associated with more severe and disabling forms of depression (Andersen and Teicher, 2008; Paus et al., 2008). For these reasons, teenagers are commonly prescribed with the antidepressant Prozac [i.e., fluoxetine (FLX)], a serotonin reuptake inhibitor (SSRI) that increases serotonin (5-HT) levels by blocking the serotonin transporter (SERT) (Nestler et al., 2002; Ansorge et al., 2007). However, the enduring effects of adolescent SSRI exposure remain poorly understood. Modeling the adolescent phase in animals may contribute not only to the study of early-onset

Received March 15, 2014; revised April 2, 2014; accepted April 4, 2014. M.R.-C. is supported by the NSF Graduate Research Fellowship (DGE1137475).

Correspondence should be addressed to Millie Rincón-Cortés, Emotional Brain Institute, Nathan Kline Institute for Psychiatric Research, 140 Old Orangeburg Road, Orangeburg, NY 10962. E-mail: millie.rinconcortes@med.nyu.edu.

DOI:10.1523/JNEUROSCI.1046-14.2014

Copyright $\odot 2014$ the authors $\quad 0270-6474 / 14 / 346719-02 \$ 15.00 / 0$ neuropsychiatric disorders such as depression and substance abuse, but may also provide insights into the long-term impact of antidepressant administration during this period.

Adolescence is a developmental window characterized by significant brain and behavioral changes that may be adaptive, but can also be detrimental and increase susceptibility for later life psychopathology (Andersen and Teicher, 2008; Paus et al., 2008). Pharmacological interventions during early development often result in long-lasting neurobehavioral alterations (Ansorge et al., 2007). For example, adolescent FLX administration alters behavioral responses to emotionally arousing and/or stressful later life environments in rodents (Iñiguez et al., 2010). In a follow-up study to explore the neurobiological mechanisms underlying the long-lasting behavioral effects of adolescent FLX exposure, Iñiguez et al. (2014) treated rodents with either vehicle (saline) or Prozac (FLX) for $\sim 2$ weeks during the juvenile period, which roughly approximates mid-adolescence in humans (Iñiguez et al., 2014). A combination of behavioral, pharmacological, and genetic approaches were then used to assess later life outcomes.

The authors first examined the effects of adolescent FLX administration on adult responses to chronic social defeat, a psychosocial stressor that involves sub- jecting rodents to repeated bouts of social subordination and that induces depressivelike symptoms in rodents (Nestler and $\mathrm{Hy}$ man, 2010). As expected, vehicle-treated animals displayed the normal social avoidance to a target animal after experiencing social defeat. The social avoidance response was diminished in FLX-treated animals after social defeat compared with vehicle-treated animals. Second, the authors examined the effects of adolescent FLX administration on adult responses to an inescapable stressful experience in the forced swim test (FST), a measure of behavioral despair in rodents used to screen for antidepressant efficacy. Adolescent FLX administration increased the latency to immobility and reduced immobility duration in the FST during adulthood. This is similar to the behavioral effect elicited by adult FLX administration on the FST, and it suggests that adolescent FLX treatment exerts an antidepressant-like effect on the FST by promoting active coping strategies (i.e., swimming more, "giving up" later) over passive ones (i.e., floating).

Previous studies have shown that preclinical models of stress induce maladaptive alterations in brain derived neurotrophic factor (BDNF) signaling within the ventral tegmental area (VTA), an area critical in mood regulation, which can be normalized through chronic FLX administration (Krishnan et al., 2008). 
Extracellular signal-regulated kinase (ERK) $1 / 2$ is a downstream target of BDNF that is involved in mediating the detrimental effects of stress, suggesting it may contribute to the long-term effects of adolescent FLX administration. Consistent with this hypothesis, Iñiguez et al. (2014) found that adolescent FLX exposure was associated with both short- and long-term reductions in ERK2 and CREB mRNA within the VTA, although social defeat increased ERK2 mRNA expression in the VTA compared with nonstressed controls. These findings suggest that adolescent FLX treatment reduces ERK activity in the VTA, whereas aversive/stressful factors increase ERK activity in the VTA. Consistent with this hypothesis, pharmacological inhibition of ERK within the VTA of adult mice induced antidepressant effects in the FST (Iñiguez et al., 2014), thereby suggesting a causal relationship between ERK activity and behavioral reactivity to stressors in naive animals.

Finally, Iñiguez et al. (2014) used viral vectors to increase or decrease ERK2 activity within the VTA to assess its role as a mediator of behavioral responses to inescapable stress (FST) in naive animals. Upregulating ERK2 levels in the VTA resulted in depressive-like behavior in the FST, as indexed by reduced latency to immobility and increased immobility compared with controls, whereas downregulating ERK2 levels had the opposite effect on both parameters and mimicked the antidepressant-like profile observed in adult rodents after adolescent FLX exposure. Collectively, these data support the notion that ERK2 signaling within the VTA regulates behavioral responses in the FST and highlights a novel role for decreased ERK2 activity within the adolescent VTA in regulating the antidepressant effects of FLX and the stress sequelae.

Depression is a complex disorder that is difficult to model in laboratory animals, as it is impossible to say whether a rodent is depressed or not. Therefore, researchers must rely on animal models in which experiences thought to trigger depression in humans (e.g., stress) produce behaviors seen in depression (such as social avoidance) that are reduced by known antidepressants (e.g., SSRIs). Iñiguez et al. (2014) integrated these important aspects of depression as well as incorporating the use of both physical and psychosocial stressors. Importantly, the researchers used chronic instead of acute FLX administration, which is of clinical relevance given that acute FLX administration exerts effects in some animal models of depression, but only prolonged FLX treatment relieves depression in humans (Nestler et al., 2002; Berton and Nestler, 2006). Furthermore, FLX was administered during a developmental period that is roughly analogous to adolescence in humans, which closely resembles the treatment regime in the clinical population of interest (teenagers). Thus, these findings may provide important insights into the relationship between developmental timing of FLX administration, antidepressant efficacy, and later life neurobehavioral outcomes-highlighting ERK signaling within the VTA as a potential mediator of these effects.

In their discussion, Iñiguez et al. (2014) suggest that these findings may aid in the development of new therapies and/or intervention strategies for the treatment of mood-related disorders among adolescents. However, it is important to note that the onset of adolescent depression is typically associated with a history of early life trauma, meaning that adolescent depression is a common maladaptive outcome resulting from adverse early life experiences including abuse and/or neglect (Andersen and Teicher, 2008). This has recently been modeled in rodents, and a link between increased amygdala activity and expression of adolescent depressive-like behavior in the FST has been identified (Raineki et al., 2012). Within this context, it would be interesting to see how infant trauma, as modeled by early life stress, maternal deprivation, or abusive attachment learning paradigms in rodents, would interact with adolescent FLX exposure in shaping adult neurobehavioral responses to aversive stimuli. Regardless, the findings reported by Iñiguez et al. (2014) have clinical relevance because antidepressants are used to treat a wide range of symptoms related to psychopathology, including anxiety disorders, post-traumatic stress disorder (PTSD), and obsessive-compulsive disorder (OCD) (Nestler et al., 2002).

Finally, it is important to remember that although there are multiple theories regarding the origins and etiology of depression, no single theory explains all the symptoms of clinical depression. Moreover, considering that the aversive stimuli used by Iñiguez et al. (2014) and in other studies are known to the activate the hypothalamic-pituitary-adrenal (HPA) axis, that the VTA is responsive to stressors, and that HPA axis dysregulation in some depressed individuals can be corrected with antidepressant administration (Nestler et al., 2002), it would be interest- ing to assess how the HPA axis (as well as the influence of adolescent FLX exposure on the developing HPA axis) may contribute to the effects seen in the study by Iñiguez et al. (2014). Is it possible that adolescent FLX administration exerts its later life effects by influencing stress reactivity and/or neurotransmission mediated by stress-associated hormones? These types of studies are important because they could reconcile neurotrophic models of depression with other models (HPA, mesolimbic) and pave the way toward a more unifying theory of the underlying mechanisms contributing to depression, thereby enabling better and more comprehensive strategies for the treatment of depression.

\section{References}

Andersen SL, Teicher MH (2008) Stress, sensitive periods and maturational events in adolescent depression. Trends Neurosci 31:183-191. CrossRef Medline

Ansorge MS, Hen R, Gingrich JA (2007) Neurodevelopmental origins of depressive disorders. Curr Opin Pharmacol 7:8-17. CrossRef Medline

Berton O, Nestler EJ (2006) New approaches to antidepressant drug discovery: beyond monoamines. Nat Rev Neurosci 7:137-151. CrossRef Medline

Iñiguez SD, Warren BL, Bolaños-Guzman CA (2010) Short- and long-term functional consequences of fluoxetine exposure during adolescence in male rats. Biol Psychiatry 67: 1057-1066. CrossRef Medline

Iñiguez SD, Alcantara LF, Warren BL, Riggs LM, Parise EM, Vialou V, Wright KN, Dayrit G, Nieto SJ, Wilkinson MB, Lobo MK, Neve RL, Nestler EJ, Bolaños-Guzman CA (2014) Fluoxetine exposure during adolescence alters responses to aversive stimuli in adulthood. J Neurosci 34:1007-1021. CrossRef Medline

Krishnan V, Han MH, Mazei-Robison M, Iñiguez SD, Ables JL, Vialou V, Berton O, Ghose S, Covington $\mathrm{HE}$ 3rd, Wiley MD, Henderson RP, Neve RL, Eisch AJ, Tamminga CA, Russo SJ, Bolaños CA, Nestler EJ (2008) AKT signaling within the ventral tegmental area regulates cellular and behavioral responses to stressful stimuli. Biol Psychiatry 64:691-700. CrossRef Medline

Nestler EJ, Hyman SE (2010) Animal models of neuropsychiatric disorders. Nat Neurosci 13: 1161-1169. CrossRef Medline

Nestler EJ, Barrot M, DiLeone RJ, Eisch AJ, Gold SJ, Monteggia LM (2002) Neurobiology of depression. Neuron 34:13-25. CrossRef Medline

Paus T, Keshavan M, Giedd JN (2008) Why do many psychiatric disorders emerge during adolescence? Nat Rev Neurosci 9:947-957. CrossRef Medline

Raineki C, Cortés MR, Belnoue L, Sullivan RM (2012) Effects of early-life abuse differ across development: infant social behavior deficits are followed by adolescent depressive-like behaviors mediated by the amygdala. J Neurosci 32:7758-7765. CrossRef Medline 